\title{
New Vaccines on the Horizon
}

\author{
Jeannette L. Comeau ${ }^{1,3} \cdot$ Jocelyn Chan $^{1} \cdot$ Kristine K. Macartney $^{1,2,3}$
}

Published online: 11 July 2016

(C) Springer Science + Business Media New York 2016

\begin{abstract}
Purpose of Review Well-established as a powerful tool for preventing disease, vaccines have immeasurably impacted health and disease epidemiology worldwide. In the era of rational vaccine design and ever-evolving technology, vaccine development and delivery is poised to improve the ability to target a range of new diseases and to improve disease prevention, in even the most remote communities worldwide.

Recent Findings New vaccines against influenza, human papillomavirus and meningococcal diseases have focused on targeting an increased number of serotypes and/or improving immunogenicity, while new vaccines against dengue and malaria are closer to being delivered to communities at need. Alternative models of protection, for example, expanded use of maternal vaccination, are also being explored and may prove effective against new diseases, such as respiratory syncytial virus. Further on the horizon are better vaccines against tuberculosis and also new vaccines for HIV, Group B Streptococcus, Group A Streptococcus, Staphylococcus aureus and cytomegalovirus. Emerging infectious diseases, including ebola and
\end{abstract}

This article is part of the Topical collection on Infectious Diseases.

Jeannette L. Comeau

jeannette.comeau@health.nsw.gov.au

1 National Centre for Immunisation Research and Surveillance (NCIRS), Kids Research Institute, The Children's Hospital at Westmead, Locked Bag 4001, Westmead, NSW 2145, Australia

2 Discipline of Paediatrics and Child Health, University of Sydney, Sydney, NSW, Australia

3 The Children's Hospital at Westmead, Sydney, NSW, Australia zika virus, present challenges for the traditional bench to bedside timeline of vaccine research, development, and deployment, proving that acceleration of this process is possible.

Summary This review covers new vaccines against the diseases above, and also briefly touches on continued efforts to ensuring life-saving immunisation is provided to all.

Keywords Vaccine - Immunisation · Pediatrics · Infectious Diseases · Vaccine development - Vaccine delivery

\section{Introduction}

Vaccines were described in 2007 by the British Medical Journal as one of the great medical milestones in the preceding 160 years [1]. While quantification of their impact is near impossible, a United States Centre for Disease Control (CDC) report based on modelling estimated vaccines prevented 322 million disease episodes in American children between 1994 and 2013 [2]. Although the triumphs of current vaccines are many, challenges in ensuring they are deployed to areas of greatest need and in developing new vaccines remain [3*0]. This review aims to update clinicians on new immunisation strategies under development and in early use to prevent childhood diseases. We focus on major pediatric pathogens, and briefly review current strategies in vaccine development, delivery and safety assessment. While it is not possible to focus on all vaccines, we aim to discuss those with the greatest implications for child health around the globe. 


\section{New Approaches to Vaccine Development and Deployment}

\section{Deploying Vaccines to Areas of Greatest Need}

The Global Alliance for Vaccines and Immunisation (GAVI) recently estimated that every year more than 1.5 million children die from vaccine preventable diseases [3*0]. In December 2010, global health leaders committed to making the next 10 years the Decade of vaccines (DOV) - to ensure discovery, development, and delivery of life-saving vaccines globally, especially to the poorest countries [4]. The four objectives of the DOV are as follows: intensified research and development for approximately 20 vaccines; advocacy and political will to mobilise donors; increased compliance and shouldering of responsibilities by developing countries; and expanded efforts in communicating the benefits of vaccines. Halfway into this initiative, a number of targeted vaccines (discussed below) have become available, with more progress to come.

\section{Who to Vaccinate?}

The target age group for pediatric vaccines has traditionally been young children, however, for certain diseases immunising pregnant women, neonates or adolescents is of increasing interest. Vaccination during pregnancy has been utilised since the 1960s as an effective tool for the prevention of maternal and neonatal tetanus [5]. Active transfer of antibodies ( $\operatorname{IgG})$ across the placenta occurs from the second trimester onwards and provides 'passive' immunisation of the infant for up to 6 months against diseases which cause high morbidity and mortality in this time frame. Protection has been shown in multiple studies for influenza vaccine, including in randomised controlled trials in pregnant women in Bangladesh and South Africa (HIV positive and negative) where infants were protected against laboratory-confirmed influenza [6, 7]. Maternal vaccination against pertussis during the early third trimester has also recently been shown to be both efficacious and safe in preventing disease in infants in the first 3 months of life [8-11]. Conducting vaccine clinical trials in pregnant women has been challenging; however, efforts to improve clinical trial and regulatory approval processes are being explored [12, 13]. Factors such as potential blunting of infant immune responses to subsequent routine immunisations and safety of both mother and infant require careful assessment [14].

Neonates are highly vulnerable to many of the diseases against which vaccines are available, but because of immune immaturity and circulating maternal antibodies vaccine administration shortly after birth is typically not protective [15]. Nonetheless, exploration of the immunogenicity, safety and efficacy of new candidate vaccines given in the first week of life is ongoing [15]. Examples include use of a live-attenuated rotavirus vaccine developed from an immunogenic neonatal strain, RV3 [16] and an acellular pertussis vaccine, shown to be safe and immunogenic in early phase 2 study [17]. The last decade has also seen an increased focus on early adolescence as a vaccine target age group, either for provision of booster doses against diseases such as pertussis, because of waning immunity to acellular pertussis vaccines given in early childhood, or to protect against diseases such as human papillomavirus (HPV). For HPV and hepatitis B vaccines, adolescents have been shown to have a robust immune response to fewer doses of vaccines than young adults.

\section{New Vaccine Technologies}

Many current vaccines were developed using 'classical vaccinology' approaches using either killed or live-attenuated pathogens, or modified toxins $[3 \cdot \bullet, 18]$. However, rapid progress in virology, genetics, biology, and biotechnology has led to novel vaccine approaches. For example, understanding the role of dendritic cells in presenting antigens to the immune system and the immune response to adjuvants has been critical. Adjuvants are added to vaccine antigens to increase the magnitude of an immune response and increasingly, they can be designed to influence the particular type of immune response elicited [19]. Aluminium-based adjuvants have been used since the 1920s to enhance antibody responses to killed inactivated or subunit vaccines [20]. There are currently over 30 adjuvants that are in use or under evaluation in various vaccines [21], including oil-in-water emulsions (for example ASO3 and MF59 in HPV and influenza vaccines, respectively), and a liposome-based adjuvant, AS01, in the recently licensed RTS,S/AS01 malaria vaccine [22].

Alternative vaccine delivery approaches are also being considered. Intradermal, inhaled, nasal or oral administration can be efficacious, but not all vaccines are amenable to these delivery modes. For example, intranasal administration of the live-attenuated influenza vaccine has proven efficacious in children (who are influenza naïve, thus allowing replication of the live-attenuated viruses), but not adults [23]. New technologies include cutaneous microneedle patches with freeze dried antigen coatings that could be used in developing countries where transportation and storage of vaccines are problematic [24, 25], and jet injection [26], a method used in hormone delivery, that may also prove viable for vaccines. Ongoing research and development as well as trials to evaluate efficacy and safety are necessary. 


\section{Vaccine Safety and Hesitancy}

Over the past 25 years, the number of vaccines on routine childhood immunisation schedules in the United States, Canada, Australia, and Europe has at least doubled. Yet during this time, it has also become evident that public confidence and trust in immunisations is fragile and requires attention [4]. Changing immunisation schedules, conflicting messages in the media and online, and ironically, the success of vaccines in reducing the visibility of serious childhood diseases, have been a threat to vaccine uptake. In some cases, vaccine hesitancy has led to failure to implement new vaccines, caused programs to be suspended, or resulted in low coverage [27]. As one of very few medical interventions that are undertaken in healthy children, ensuring safety and communicating this to parents is paramount.

In 2011 the World Health Organization (WHO) and partners developed the Global Vaccine Safety Blueprint, a document aimed at ensuring that each country has a mechanism to evaluate and ensure the safety of vaccines [28]. The Global vaccine safety initiative (GVSI) was created to implement the eight Blueprint objectives, which include Adverse events following immunisation (AEFI) detection, vaccine safety communication, and global analysis and response. Within two years of its inception, 50 countries are involved in the GVSI, accounting for approximately $77 \%$ of the world's population [29]. Global coordination of AEFI monitoring, as well as communication between regulatory bodies, allow early detection of adverse events that can prompt investigation. This is essential in the current climate of increasingly complex immunisation schedules. Clinicaltrials.gov is an online resource that enables the public to have access to information on vaccine and many other clinical trials. Maintained by the National Library for Medicine (NLM) at the National Institute for Health (NIH) in the USA, medical trials in human volunteers occurring in 193 countries are recorded. As of May 6, 2016, information on 6016 vaccine trials was recorded, 2159 of which were in pediatrics (children aged 0-17 years); trials recorded on this register by disease are shown in Table 1 .

\section{Specific Diseases}

\section{Dengue}

Dengue virus, a flavivirus spread by the Aedes species of mosquito, infects at least 390 million people per year [30]. It has been estimated that up to $95 \%$ of cases occur in children $<15$ years of age [31], with varying clinical presentations. There are four dengue serotypes and as only type-specific infection gives lifelong immunity, infection up to four times in possible. In addition, repeat infections present a much higher risk of complications and shock [32]. There is no specific treatment and vector control efforts have been ineffective to date [30].

In 2015, a live recombinant tetravalent chimeric dengue vaccine, with antigens from all four dengue serotypes substituted into a yellow fever vaccine backbone (CYDTDV) [33], became the first ever licensed dengue vaccine. It was evaluated in two major phase III clinical trials, in Asia among children 2-4 years, and in Latin America in children 9-16 years. The overall vaccine efficacy from these studies was 59.2\% (95\% CI 52.3-65.0) against any of the four serotypes of dengue; however, serotype-specific efficacy for dengue serotype two virus was poor [34]. Immune responses and vaccine efficacy were also greater in children who were flavivirus seropositive (indicating at least one previous infection), suggesting that the vaccine will be most useful in endemic countries [34-36, 37•]. Although modelling studies have demonstrated that even partially efficacious vaccines can lead to significant disease reduction [38], these issues are complex when considering how to deploy the vaccine. The WHO Strategic advisory group of experts (SAGE) on Immunisation is currently developing recommendations for use of CYD-TDV [39], but in the meantime, one endemic country, the Philippines, began mass vaccination in April 2016 [40].

A number of other dengue vaccines are currently in development. DENVax, a mixture of chimeric DENV1, DENV3 and DENV4 antigens on a DENV2 backbone plus a whole live-attenuated DENV2 component [41], is in Phase II studies in Colombia, Puerto Rico, Singapore and Thailand [42, 43]. Other vaccine candidates are tetravalent admixtures of monovalent live-attenuated vaccine strains covering DENV1-4 (TV003 and TV005). First tested individually and then in combination, trials have demonstrated safety and immunogenicity to 6 months [44] with phase II studies currently underway in Thailand and Brazil $[45,46]$.

\section{Malaria}

Despite widespread use of insecticide impregnated bed nets, insecticide spraying, and malaria treatment, morbidity and mortality remain high. In 2015, there were an estimated 438,000 deaths due to malaria, with $70 \%$ occurring in children $<5$ years of age [47].

The complexities of the parasite lifecycle make vaccine development difficult. Most efforts to date have focused on Plasmodium falciparum, which is responsible for more than $98 \%$ of malaria mortality [48]. In July 2015, the European Medicines Agency, approved the first malaria vaccine, known as RTS,S/AS01 (Mosquirix ${ }^{\mathrm{TM}}$, GSK), for 
Table 1 Number of vaccine clinical trials currently registered on clinicaltrials.gov for specified diseases. (Current as of 6 May 2016)

\begin{tabular}{|c|c|c|c|c|c|c|c|c|c|}
\hline \multirow[t]{2}{*}{ Disease } & \multicolumn{2}{|c|}{$\mathrm{N}$ Trials } & \multicolumn{2}{|c|}{$\begin{array}{l}\text { Phase III \& } \\
\text { IV }\end{array}$} & \multicolumn{2}{|c|}{$\begin{array}{l}\text { Completed with } \\
\text { results }\end{array}$} & \multicolumn{2}{|c|}{$\begin{array}{l}\text { Completed with results phase } \\
\text { III \& IV }\end{array}$} & \multirow[t]{2}{*}{ Vaccine currently available } \\
\hline & All & Peds & All & Peds & All & Peds & All & Peds & \\
\hline All vaccine clinical trials & 6061 & 2159 & 1815 & 1042 & 976 & 490 & 524 & 326 & - \\
\hline CMV & 36 & 4 & 5 & 0 & 1 & 0 & 0 & 0 & No \\
\hline Dengue virus & 83 & 27 & 8 & 7 & 2 & 1 & 0 & 0 & Yes \\
\hline Ebola virus & 45 & 5 & 6 & 2 & 0 & 0 & 0 & 0 & No \\
\hline GAS & 18 & 2 & 0 & 0 & 4 & 0 & 0 & 0 & No \\
\hline GBS & 15 & 0 & 0 & 0 & 4 & 0 & 0 & 0 & No \\
\hline $\mathrm{HIV}^{\dagger}$ & 576 & 113 & 72 & 20 & 43 & 14 & 5 & 3 & No \\
\hline HPV & 233 & 134 & 99 & 68 & 74 & 46 & 48 & 31 & Yes \\
\hline Influenza & 1366 & 491 & 589 & 274 & 318 & 135 & 187 & 94 & Yes \\
\hline Malaria & 172 & 53 & 12 & 12 & 7 & 7 & 0 & 0 & No \\
\hline Meningococcus & 91 & 80 & 53 & 48 & 32 & 30 & 15 & 13 & Yes \\
\hline Rotavirus & 127 & 120 & 58 & 58 & 32 & 31 & 22 & 22 & Yes \\
\hline RSV & 38 & 15 & 3 & 1 & 3 & 3 & 0 & 0 & No* \\
\hline Staphylococcus aureus & 24 & 0 & 8 & 0 & 5 & 0 & 0 & 0 & No \\
\hline Tuberculosis & 96 & 22 & 8 & 2 & 5 & 2 & 0 & 0 & Yes (BCG) \\
\hline Zika virus & 0 & 0 & 0 & 0 & 0 & 0 & 0 & 0 & No \\
\hline
\end{tabular}

* Passive immunisation via monoclonal antibody infusion (Palivizumab)

$\dagger$ Includes clinical trials of HIV vaccines and other vaccines in HIV-infected population

$C M V$ Cytomegalovirus, GAS Group A Streptococcus, GBS Group B Streptococcus, HIV Human immunodeficiency virus, HPV Human papillomavirus, $R S V$ Respiratory syncytial virus, $B C G$ Bacillus Calmette-Guérin

use outside of Europe. The vaccine was developed in partnership between GSK and the PATH Malaria vaccine initiative, with support from the Bill and Melinda Gates Foundation, representing an innovative new funding model and public-private partnership. It is a recombinant vaccine, where a sporozoite protein is fused with HBsAg inducing a specific immune response to prevent blood stage infection. Phase III studies using a three dose schedule and a $4^{\text {th }}$ booster dose were conducted in seven African countries [49]. Efficacy against malaria 14 months after vaccination was $50.4 \%$ (95\% CI 45.8-54.6) in children immunised at 5-17 months, but lower in infants immunised at 6-12 weeks of age (30.1\% [95\% CI 23.6-36.1]). Efficacy waned thereafter and an increased risk of severe malaria among vaccinated children occurred in the last 27 months of the study. This suggests the need for a 4-dose schedule starting from 5-6 months of age. Given the complexities involved, WHO recommended pilot implementation in 3-5 subnational sub-Saharan areas before considering wider country level introduction [50]. Other vaccines being trialled include three promising candidates using whole sporozoites [51-53] and a heterologous 'prime boost' strategy [54, 55]. Interesting "transmission-blocking" vaccines target sexual erythrocytic and early mosquito stage antigens, as the parasite passes from human host to mosquito and aim to reduce burden of disease by reducing transmission [56, 57].

\section{Tuberculosis and Human Immunodeficiency Virus (HIV) Vaccines}

Tuberculosis (TB) remains a significant cause of child morbidity and mortality worldwide, with an estimated 140 000 deaths in children worldwide in 2014 [58]. The emergence of drug resistant Mycobacterium tuberculosis strains and prevalence of HIV co-infection has further complicated management [59]. The live-attenuated bacillus calmette-guérin $(\mathrm{BCG})$ vaccine is the only licensed $\mathrm{TB}$ vaccine and has some effectiveness at preventing miliary and meningeal TB in children [60, 61]. However, BCG has limited effectiveness in older children and adults, and against pulmonary TB, and carries a small risk of severe complications including disseminated BCG infection [62], which outweighs potential benefits in countries with low TB prevalence [63]. Recently supply has been threatened by a global shortage [64]. A limited understanding of the specific human immune response to $M$. tuberculosis infection and a lack of immunological correlates of 
protection that can predict vaccine efficacy in humans with certainty remain challenges in vaccine development [65].

TB vaccine candidates in development (approximately 13) are broadly aimed either at replacing BCG or boosting the immune response in already infected persons, and include whole cell derived, viral vectored or adjuvanted protein subunit vaccines. For example, in South Africa, one recombinant BCG vaccine candidate (VPM1002) has been evaluated through a phase II study in newborn children with results awaited [66], and a candidate live-attenuated mycobacterium tuberculosis vaccine is entering into a phase $1 \mathrm{~b}$ trial of safety and immunogenicity in healthy neonates [67].

Despite over thirty years of vaccine research following the isolation of HIV in 1983, no candidates are close to prophylactic use in humans [68]. However, much has been learnt about the immunopathogenesis of the virus, informing vaccine development efforts. One vaccine candidate that demonstrated limited efficacy against HIV-1 in adults in Thailand [69], is being further investigated. Only one pediatric trial, that has not begun recruitment, is registered on clinicaltrials.gov, utilising a killed HIV-1 viral particle vaccine (REMUNE ${ }^{\mathrm{TM}}$, BioPharma) [70].

\section{Influenza}

Seasonal epidemics of influenza occur globally with an annual attack rate estimated at 20-30\% in children [71]. Current vaccines, while moderately effective in all ages $>6$ months, induce strain specific immunity and have to be updated yearly because of antigenic drift [72]. They include injected inactivated influenza vaccines (IIV), and a nasally administered live-attenuated influenza vaccine (LAIV) [72], which have 56-64\%, and up to $80 \%$ efficacy, respectively [73]. New quadrivalent influenza virus (QIV) vaccines (both as IIV and LAIV) contain a second influenza $B$ strain in addition to the two influenza $A$ and single B lineage in the trivalent, and will offer greater protection in years in which both or a mismatched B lineages circulate. QIVs are being incorporated into programs worldwide. The MF59 adjuvanted seasonal vaccine (e.g. Fluad $^{\circledR}$, Novartis) has been licensed for the elderly population but has had limited use in pediatrics [74].

Strategies to advance the speed and scale of influenza vaccine production include recombinant vaccines, the use of virus like particles, DNA vaccines, and virus-vectored vaccines [72]. The trivalent seasonal recombinant haemagglutinin vaccine (e.g. Flublok ${ }^{\circledR}$, Protein Sciences) produced in insect cells has already been licensed in the USA [72]. Progress is also being made towards the development of a universal influenza virus vaccine [75], a major milestone facilitated by identification of neutralising antibodies to the conserved parts of the haemagglutinin protein, including the stalk. Clinical trials to test this hypothesis have been initiated [75].

\section{Respiratory Syncytial Virus (RSV)}

RSV was initially isolated in 1957 [76], and the quest for a vaccine has been underway since. It remains the most common acute lower respiratory tract illness in children $<5$ years of age worldwide, accounting for 33.8 million cases annually, and 3.4 million hospitalisations [77], with infants most severely affected.

A candidate formalin-inactivated RSV vaccine in the 1960s paradoxically resulted in more severe disease in a phase 3 clinical trial [78, 79], setting back progress on vaccine development for decades. Passive immunisation of high risk infants via monthly infusions of RSV-specific immunoglobulin (Palivizumab) has some efficacy, but is limited by cost and complexity [80]. Vaccine development is now focused on either maternal vaccination to protect infants in the first months of life (another form of 'passive immunisation') or active immunisation of infants and young children. Better understanding of RSV immunopathogenesis, and particularly delineation of the antigenic epitopes of the RSV F (fusion) protein responsible for entry into the host cell [81•], has led to a resurgence in vaccine development, with 16 vaccine candidates in active clinical trials (including two in Phase III studies) by December 2015 [82, 83]. Current vaccine candidates include RSV F nanoparticle vaccines, live-attenuated, particle-based, subunit-based and genebased vectors [83].

Two live intranasal RSV vaccine candidates (MEDI-534 and -559) showed immunogenicity in a Phase 1 clinical trials in young children $[84,85]$. Additionally, in a Phase II study in 330 non-pregnant women of childbearing age a 2-dose recombinant RSV fusion protein nanoparticle vaccine (RSV F vaccine) candidate was safe and immunogenic [86]. Post-hoc analysis demonstrated reduction in RSV infections in these women by $50 \%$ over the following 112 days. Studies in pregnant women are currently in recruitment phase [87].

\section{Human Papillomavirus (HPV)}

Discovery of HPV's central role in the development of cervical and anogenital cancers, as well as approximately $60 \%$ of head and neck cancers, prompted development of a vaccine against HPV; the second cancer preventing vaccine after hepatitis $B$. The first two HPV vaccines have been available for a decade and include oncogenic HPV genotypes 16 and 18, responsible for approximately $70 \%$ of cervical cancer worldwide with the quadrivalent vaccine also including non-oncogenic types 6 and 11, responsible for external genital lesions (warts) [88, 89]. Both vaccines 
have now shown efficacy in prevention of pre-cancerous lesions in males and females [90, 91•, 92, 93] and effectiveness in preventing high grade cervical lesions [94, 95] and genital warts [96, 97]. While continued progress is needed on vaccine implementation in low-middle income countries where cervical screening programs are not well established, a next generation 9-valent HPV vaccine has recently been licensed. It contains 5 additional oncogenic HPV types and is estimated to prevent approximately $90 \%$ of all cervical cancers [91, 98]. The safety profile of this 9-valent vaccine appears similar to the quadrivalent, but incremental cost effectiveness may limit uptake.

Another major development in HPV vaccination has been evidence supporting the move from 3- to 2-dose schedules in young adolescent females and males. Those aged 9-15 years who receive two vaccine doses 6 months apart, have a comparable immune response to older persons (16-26 years) given three vaccines doses over the same time course. This schedule is now recommended by the WHO for HPV vaccination of girls in this age group [99], and will hopefully improve acceptability and uptake.

\section{Meningococcus}

Neisseria meningiditis, a common coloniser of the nasopharynx, can cause rapidly evolving meningitis or meningococcemia. There are 12 known serotypes; vaccines now exist against the five most common (A, C, Y, W-135, and B) disease causing strains. The first polysaccharide vaccines were effective in outbreak scenarios, but did not reduce nasal carriage or provide long-term immunity, and were ineffective in young children [100, 101]. Following the success of protein-polysaccharide conjugated $\mathrm{He}$ mophilius influenza type $B$ vaccines, conjugated meningococcal vaccines against serotype $\mathrm{A}$ or $\mathrm{C}$ alone, or A,C,Y,W-135 together were developed. These vaccines have demonstrated a major impact on serotype specific invasive meningococcal disease (particularly serotype $\mathrm{C}$ disease) when used in population-based programs [102, 103]. Country-specific recommendations are highly variable, depending on the local disease epidemiology, serotype prevalence and individual risk factors (e.g. underlying immunodeficiency). The recent introduction of a low-cost type A conjugate vaccine in the Sub-Saharan African 'meningitis belt' has had a major impact in that region, with a $94 \%$ decrease in invasive meningococcal disease incidence following vaccination of 1.8 million people in a 10-day period in Chad [104].

Development of a type B vaccine proved highly challenging, because of capsular polysaccharide homology with polysilic acid structures present on human neural cells [105]. Two recent serogroup B vaccines used 'reverse vaccinology', where the sequenced genome was scanned for the most universally applicable antigen candidates, which were then manufactured using recombinant technology and tested in prototype vaccines. A four-antigen component vaccine (Bexsero ${ }^{\circledR}$, GSK, previously Novartis) is now licensed for use in a four dose schedule in infants (or two doses in older children and adults), but population level programs have only been implemented in one community in Canada since May 2014 and country-wide in the UK since November 2015 [106, 107]. Vaccine cost effectiveness and the need to use prophylactic paracetamol to prevent post vaccination fever in infants have been factors influencing the decision not to publicly fund the vaccine in other countries where meningococcal B disease is prevalent, such as Australia [108]. The bivalent recombinant vaccine, rLP2086 (Trumenba ${ }^{\circledR}$, Pfizer), has also been approved in the USA for adolescents [109].

\section{Other Vaccines}

Many vaccine candidates against common pediatric pathogens are in the developmental pipeline; including against Group B Streptococcus (GBS, Streptococcus agalactia), Group A Streptococcus (GAS, Streptococcus pyogenes), S. aureus and cytomegalovirus (CMV). Vaccines against ebola and zika virus infection have also been a focus of development after recent outbreaks.

Maternal vaccination against GBS was proposed approximately 40 years ago [110]. However, only 4 of 15 currently registered clinical trials have reported results. A multi-national phase II study reported safety and immunogenicity of a trivalent GBS vaccine administered to pregnant women; antibodies were measurable in mothers, and in infants for up to 91 days [13]. GASrelated disease has been described since the 1600s [111] with great variability in manifestations. Both disease and sequelae, including acute rheumatic fever, cause considerable morbidity and mortality; a 2005 study estimated that worldwide prevalence of serious GAS disease was at least 18.1 million, with 1.78 million new cases annually [112]. There are at least 18 current vaccine candidates, with three of these in Phase 1 or 2 trials [113]. S. aureus is a ubiquitous bacteria that causes a wide range of disease from skin and soft tissue infections to endocarditis to toxin-mediated illness. The increasing prevalence of methicillin-resistant $S$. aureus and diminishing antimicrobial treatment options underlines the importance of vaccine development. However, there are currently no pediatric clinical trials; most vaccine candidates have been evaluated in high risk adult patients but with limited success.

Cytomegalovirus is the most common congenital infection, occurring in $0.5-2 \%$ of all live births but with 
a variable phenotype. CMV has been the subject of attempts at vaccine development for decades, with limited success; current candidates include live-attenuated, subunit, and vectored vaccines. Phase I and II clinical trials are underway in women of childbearing age, and would especially target those with a toddler in the home [114].

The recent catastrophic ebola outbreak in 2014-2015 in Africa accelerated vaccine research and development. There are currently 45 clinical trials of vaccine candidates, with the two main candidates being rVSV-ZEBOV, a recombinant vesicular stomatitis vaccine, and ChAd3EBO-Z, a chimpanzee adenovirus-3 vaccine. In 2015, rVSV-ZEBOV was evaluated in a cluster-randomised trial that employed 'ring' vaccination, where all near contacts of the index case are immunised. Interim results suggest both efficacy and safety [115•]. The ChAd3-EBO-Z vaccine recently completed phase $1 \mathrm{~b}$ testing in adults and was found to be safe, thus supporting moving to phase $2 / 3$ trials [116]. The emergence of zika virus in South and Central America as a new infectious cause of congenital neurologic disease, hallmarked by microcephaly, and as a trigger for Guillain-Barré syndrome resulted in the WHO declaring a global health emergency on 1 February 2016 [117]. Work on DNA-based, live-attenuated, and recombinant VSV vaccine candidates is proceeding, although gaps in knowledge around the disease itself need to be filled [118]. While this will take some time, it is possible that similar fast-tracking of clinical trials may occur as was the case with ebola vaccines.

\section{Conclusions}

Vaccine research and development is in a state of continual change and evolution. The number of infectious diseases for which regular vaccination is now offered has doubled within the past 25 years and will continue to grow. For the pediatrician, this can be readily observed by changes in clinical practice; cases of epiglottitis are now rare, and meningitis although still present, is much less common. As antimicrobial resistance continues to increase, and with new emerging infectious diseases, such as zika virus, vaccines are poised to provide an even greater impact on eliminating childhood disease.

\section{Compliance with Ethics Guidelines}

Disclosure Jeannette L Comeau, Jocelyn Chan, and Kristine K Macartney declare that they have no conflict of interest.

Human and Animal Rights and Informed Consent This article does not contain any studies with human or animal subjects performed by any of the authors.

\section{References}

Papers of particular interest, published recently, have been highlighted as:

- Of importance

-• Of major importance

1. Worboys M. Vaccines: conquering untreatable diseases. BMJ. 2007;334(Suppl 1):s19.

2. Whitney $\mathrm{CG}$, et al. Benefits from immunization during the vaccines for children program era-United States, 1994-2013. MMWR Morb Mortal Wkly Rep. 2014;63(16):352-5.

3. $\bullet$ Nabel GJ. Designing tomorrow's vaccines. N Engl J Med. 2013;368(6):551-60. Comprehensive review of current vaccine development research and development.

4. Moxon ER, Siegrist CA. The next decade of vaccines: societal and scientific challenges. Lancet. 2011;378(9788):348-59.

5. Schofield FD, Tucker VM, Westbrook GR. Neonatal tetanus in New Guinea. effect of active immunization in pregnancy. $\mathrm{Br}$ Med J. 1961;2(5255):785-9.

6. Madhi SA, et al. Influenza vaccination of pregnant women and protection of their infants. N Engl J Med. 2014;371(10):918-31.

7. Zaman K, et al. Effectiveness of maternal influenza immunization in mothers and infants. $\mathrm{N}$ Engl $\mathrm{J}$ Med. 2008;359(15):1555-64.

8. Marshall $\mathrm{H}$, et al. Vaccines in pregnancy: the dual benefit for pregnant women and infants. Hum Vaccin Immunother. 2016;12:848-56.

9. Donegan K, King B, Bryan P. Safety of pertussis vaccination in pregnant women in UK: observational study. BMJ. 2014;349:g4219.

10. Amirthalingam G, et al. Effectiveness of maternal pertussis vaccination in England: an observational study. Lancet. 2014;384(9953):1521-8.

11. Munoz FM, et al. Safety and immunogenicity of tetanus diphtheria and acellular pertussis (Tdap) immunization during pregnancy in mothers and infants: a randomized clinical trial. JAMA. 2014;311(17):1760-9.

12. Moniz MH, Beigi RH. Vaccination during pregnancy. Obstet Gynecol Surv. 2016;71(3):178-86.

13. Madhi SA, et al. Safety and immunogenicity of an investigational maternal trivalent group B streptococcus vaccine in healthy women and their infants: a randomised phase $1 \mathrm{~b} / 2$ trial. Lancet Infect Dis. 2016;. doi:10.1016/S1473-3099(16)00152-3.

14. Munoz FM, et al. Research on vaccines during pregnancy: protocol design and assessment of safety. Vaccine. 2013;31(40):4274-9.

15. Mohr E, Siegrist CA. Vaccination in early life: standing up to the challenges. Curr Opin Immunol. 2016;41:1-8.

16. Bines JE, et al. Safety and immunogenicity of RV3-BB human neonatal rotavirus vaccine administered at birth or in infancy: a randomised, double-blind, placebo-controlled trial. Lancet Infect Dis. 2015;15(12):1389-97.

17. Wood N. et al. High and persistent antibody responses to monovalent acellular pertussis vaccine at birth - a large randomised controlled trial. in ID Week. 2015. San Diego.

18. Rappuoli R, Medaglini D. Big science for vaccine development. Vaccine. 2014;32(37):4705-7.

19. Coffman RL, Sher A, Seder RA. Vaccine adjuvants: putting innate immunity to work. Immunity. 2010;33(4):492-503.

20. Lindblad EB. Aluminium compounds for use in vaccines. Immunol Cell Biol. 2004;82(5):497-505. 
21. Garcon N, Hem S, Friede M. Evolution of adjuvants across the centuries. In: Plotkin SA, Orenstein WA, Offit PA, editors. Vaccines. Amsterdam: Elsevier; 2013.

22. Gosling R, von Seidlein L. The Future of the RTS, S/AS01 malaria vaccine: an alternative development plan. PLoS Med. 2016;13(4):e1001994.

23. Osterholm MT, et al. Efficacy and effectiveness of influenza vaccines: a systematic review and meta-analysis. Lancet Infect Dis. 2012;12(1):36-44.

24. Arya, J. and M.R. Prausnitz, Microneedle patches for vaccination in developing countries. J Control Release, 2015.

25. Larraneta E, et al. Microneedles: a New Frontier in Nanomedicine Delivery. Pharm Res. 2016;33(5):1055-73.

26. Hogan NC, et al. Needle-free delivery of macromolecules through the skin using controllable jet injectors. Expert Opin Drug Deliv. 2015;12(10):1637-48.

27. Dube E, Vivion M, MacDonald NE. Vaccine hesitancy, vaccine refusal and the anti-vaccine movement: influence, impact and implications. Expert Rev Vaccines. 2015;14(1):99-117.

28. World Health Organization. WHO The global vaccine safety initiative (GVSI). 2016. http://www.who.int/vaccine_safety/ initiative/en/. Accessed cited 2016.

29. World Health Organization, Global vaccine safety initiative: 2013-2014 annual report. 2015: France.

30. Bhatt S, et al. The global distribution and burden of dengue. Nature. 2013;496(7446):504-7.

31. Bhattacharya MK, et al. Dengue: a growing menace-a snapshot of recent facts, figures \& remedies. Int $\mathrm{J}$ Biomed Sci. 2013;9(2):61-7.

32. Martina BE, Koraka P, Osterhaus AD. Dengue virus pathogenesis: an integrated view. Clin Microbiol Rev. 2009;22(4):564-81.

33. Schwartz LM, et al. The dengue vaccine pipeline: implications for the future of dengue control. Vaccine. 2015;33(29):3293-8.

34. Capeding MR, et al. Clinical efficacy and safety of a novel tetravalent dengue vaccine in healthy children in Asia: a phase 3 , randomised, observer-masked, placebo-controlled trial. The Lancet. 2014;384(9951):1358-65.

35. Dayan GH, et al. Immunogenicity and safety of a recombinant tetravalent dengue vaccine in children and adolescents ages 9-16 years in Brazil. Am J Trop Med Hyg. 2013;89(6):1058-65.

36. Hss AS, et al. Safety and immunogenicity of a tetravalent dengue vaccine in healthy children aged 2-11 years in Malaysia: a randomized, placebo-controlled. Phase III study. Vaccine. 2013;31(49):5814-21.

37. - Villar L, et al. Efficacy of a tetravalent dengue vaccine in children in Latin America. N Engl J Med. 2015;372(2):113-23. Large blinded, placebo-controlled RCT evaluating recombinant, live, attenuated, tetravalent dengue vaccine, demonstrating efficacy.

38. Rodriguez-Barraquer I, et al. Potential opportunities and perils of imperfect dengue vaccines. Vaccine. 2014;32(4):514-20.

39. World Health Organization. Dengue vaccine research. immunization, vaccines and biologicals $2016 \mathrm{http} / / / \mathrm{www}$.who.int/ immunization/research/development/dengue_vaccines/en/. Accessed 25 Jan 2015.

40. AFP. Historic dengue vaccine programme launched in the Philippines. 2016. http://www.channelnewsasia.com/news/ asiapacific/historic-dengue-vaccine/2664344.html. Accessed 28 April 2016.

41. Osorio JE, et al. Safety and immunogenicity of a recombinant live attenuated tetravalent dengue vaccine (DENVax) in flavivirus-naive healthy adults in Colombia: a randomised, placebo-controlled, phase 1 study. Lancet Infect Dis. 2014;14(9):830-8.
42. ClinicalTrials.gov. Study to investigate the safety and immunogenicity of a tetravalent chimeric dengue vaccine in health volunteers 1.5-45 years. 2011. https://clinicaltrials.gov/ ct2/show/NCT01511250.Accessed 25 Jan 2016.

43. ClinicalTrials.gov. safety and immunogenicity of different schedules of takeda's tetravalent dengue vaccine candidate (TDV) in healthy participants. 2014. https://clinicaltrials.gov/ ct2/show/NCT02302066. Accessed 26 Jan 2016.

44. Durbin AP, et al. A single dose of any of four different live attenuated tetravalent dengue vaccines is safe and immunogenic in flavivirus-naive adults: a randomized, double-blind clinical trial. J Infect Dis. 2013;207(6):957-65.

45. ClinicalTrials.gov. evaluating the safety of and immune response to a dengue vaccine (tv003) in healthy adults, adolescents, and children in Thailand. 2015. https://clinicaltrials.gov/ ct2/show/NCT02332733. Accessed 25 Jan 2016.

46. ClinicalTrials.gov. Phase II Trial to Evaluate Safety and Immunogenicity of a Dengue 1,2,3,4 (Attenuated) Vaccine. 2012. https://clinicaltrials.gov/ct2/show/NCT01696422. Accesssed 25 Jan 2015.

47. World Health Organization, World Malaria Report. 2015: Brussels.

48. Hoffman SL, et al. The march toward malaria vaccines. Vaccine. 2015;33(Suppl 4):D13-23.

49. Leach A, et al. Design of a phase III multicenter trial to evaluate the efficacy of the RTS, S/AS01 malaria vaccine in children across diverse transmission settings in Africa. Malar $\mathrm{J}$. 2011;10:224.

50. Malaria vaccine: WHO position paper-January 2016. Wkly Epidemiol Rec. 2016; 91(4):33-51.

51. Seder RA, et al. Protection against malaria by intravenous immunization with a nonreplicating sporozoite vaccine. Science. 2013;341(6152):1359-65.

52. Richie TL, et al. Progress with Plasmodium falciparum sporozoite (PfSPZ)-based malaria vaccines. Vaccine. 2015;33(52):7452-61.

53. Epstein JE, et al. Live Attenuated Malaria Vaccine Designed to Protect Through Hepatic CD8+T Cell Immunity. Science. 2011;334(6055):475-80.

54. Kimani D, et al. Translating the Immunogenicity of Prime-boost Immunization With ChAd63 and MVA ME-TRAP From Malaria Naive to Malaria-endemic Populations. Mol Ther. 2014;22(11):1992-2003.

55. Ogwang C, et al. Prime-boost vaccination with chimpanzee adenovirus and modified vaccinia Ankara encoding TRAP provides partial protection against Plasmodium falciparum infection in Kenyan adults. Sci Translat Med. 2015;7(286):286re5.

56. $\mathrm{Wu} \mathrm{Y}$, et al. Phase 1 trial of malaria transmission blocking vaccine candidates Pfs 25 and Pvs 25 formulated with montanide ISA 51. PLoS One. 2008;3(7):e2636.

57. ClinicalTrials.gov. Safety and Immunogenicity of Plant-Derived Pfs 25 VLP-FhCMB Malaria Transmission Blocking Vaccine in Healthy Adults. 201328 Jan 2016]; Available from: https:// clinicaltrials.gov/ct2/show/NCT02013687. Accessed 28 Jan 2016.

58. World Health Organization, Global tuberculosis report 20th Edition. 2015.

59. Gunther G. Multidrug-resistant and extensively drug-resistant tuberculosis: a review of current concepts and future challenges. Clin Med (Lond). 2014;14(3):279-85.

60. Rodrigues LC, Diwan VK, Wheeler JG. Protective effect of BCG against tuberculous meningitis and miliary tuberculosis: a meta-analysis. Int J Epidemiol. 1993;22(6):1154-8.

61. Trunz BB, Fine P, Dye C. Effect of BCG vaccination on childhood tuberculous meningitis and miliary tuberculosis 
worldwide: a meta-analysis and assessment of cost-effectiveness. Lancet. 2006;367(9517):1173-80.

62. Hesseling AC, et al. The risk of disseminated Bacille CalmetteGuerin (BCG) disease in HIV-infected children. Vaccine. 2007;25(1):14-8.

63. Manissero D, et al. Assessing the impact of different BCG vaccination strategies on severe childhood TB in low-intermediate prevalence settings. Vaccine. 2008;26(18):2253-9.

64. Marais BJ, et al. Interrupted BCG vaccination is a major threat to global child health. Lancet Respir Med. 2016;4:251-3.

65. Nunes-Alves $\mathrm{C}$, et al. In search of a new paradigm for protective immunity to TB. Nat Rev Microbiol. 2014;12(4):289-99.

66. Principi N, Esposito S. The present and future of tuberculosis vaccinations. Tuberculosis (Edinb). 2015;95(1):6-13.

67. Spertini F, et al. Safety of human immunisation with a liveattenuated Mycobacterium tuberculosis vaccine: a randomised, double-blind, controlled phase I trial. The Lancet Respiratory Medicine. 2015;3(12):953-62.

68. Fischinger PJ, et al. Current status and strategies for vaccines against diseases induced by human T-cell lymphotropic retroviruses (HTLV-I,-II,-III). Cancer Res. 1985;45(9 Suppl):4694s-9s.

69. Rerks-Ngarm S, et al. Vaccination with ALVAC and AIDSVAX to prevent HIV-1 infection in Thailand. $\mathrm{N}$ Engl $\mathrm{J}$ Med. 2009;361(23):2209-20.

70. ClinicalTrials.gov. REMUNE HIV/AIDS vaccine phase ii pediatric safety \& efficacy clinical study. 2014. https:// clinicaltrials.gov/ct2/show/NCT02291809. Accessed 28 April 2016.

71. World Health Organization. Influenza (Seasonal) fact sheet no. 211. 2014. http://www.who.int/mediacentre/factsheets/fs211/en/ . Accessed 28 Jan 2016.

72. Krammer F, Palese P. Advances in the development of influenza virus vaccines. Nat Rev Drug Discov. 2015;14(3):167-82.

73. Jefferson $\mathrm{T}$, et al. Vaccines for preventing influenza in healthy children. Cochrane Database Syst Rev. 2012;8:CD004879.

74. Tinoco JC, et al. Immunogenicity, reactogenicity, and safety of inactivated quadrivalent influenza vaccine candidate versus inactivated trivalent influenza vaccine in healthy adults aged $>/$ $=18$ years: a phase III, randomized trial. Vaccine. 2014;32(13):1480-7.

75. Berlanda Scorza F, Tsvetnitsky V, Donnelly JJ. Universal influenza vaccines: shifting to better vaccines. Vaccine. 2016;34:2926-33.

76. Chanock R, Roizman B, Myers R. Recovery from infants with respiratory illness of a virus related to chimpanzee coryza agent (CCA). I. Isolation, properties and characterization. Am J Hyg. 1957;66(3):281-90.

77. Nair H, et al. Global burden of acute lower respiratory infections due to respiratory syncytial virus in young children: a systematic review and meta-analysis. Lancet. 2010;375(9725):1545-55.

78. Kim HW, et al. Respiratory syncytial virus disease in infants despite prior administration of antigenic inactivated vaccine. Am J Epidemiol. 1969;89(4):422-34.

79. Kapikian AZ, et al. An epidemiologic study of altered clinical reactivity to respiratory syncytial (RS) virus infection in children previously vaccinated with an inactivated RS virus vaccine. Am J Epidemiol. 1969;89(4):405-21.

80. Higgins D, Trujillo C. Keech C Advances in RSV vaccine research and development-a global agenda. Vaccine. 2016;34:2870-5.

81. McLellan JS, et al. Structure-based design of a fusion glycoprotein vaccine for respiratory syncytial virus. Science. 2013;342(6158):592-8. Research article outlining structurebased vaccine strategy and targeted engineering for vaccine development, specifically $R S V F$ protein in this case.
82. Modjarrad K, et al. WHO consultation on respiratory syncytial virus vaccine development report from a World health organization Meeting held on 23-24 March 2015. Vaccine. 2016;34(2):190-7.

83. PATH. RSV Vaccine Snapshot. 2015. http://www.path.org/ vaccineresources/details.php?i=1562. Accessed 19 Apr 2016.

84. Malkin E, et al. Safety and immunogenicity of a live attenuated RSV vaccine in healthy RSV-seronegative children 5 to 24 months of age. PLoS One. 2013;8(10):e77104.

85. Yang CF, et al. Implication of respiratory syncytial virus (RSV) $F$ transgene sequence heterogeneity observed in Phase 1 evaluation of MEDI-534, a live attenuated parainfluenza type 3 vectored RSV vaccine. Vaccine. 2013;31(26):2822-7.

86. Glenn GM, et al. A randomized, blinded, controlled, doseranging study of a respiratory syncytial virus recombinant fusion (F) nanoparticle vaccine in healthy women of childbearing age. J Infect Dis. 2016;213(3):411-22.

87. ClinicalTrials.gov. a study to determine the Safety and efficacy of the RSV F vaccine to protect infants via maternal immunization. 2015. https://clinicaltrials.gov/ct2/show/ NCT02624947. Accessed 28 April 2016.

88. Georgousakis M, et al. Population-wide vaccination against human papillomavirus in adolescent boys: australia as a case study. Lancet Infect Dis. 2012;12(8):627-34.

89. Schmeler KM, Sturgis EM. Expanding the benefits of HPV vaccination to boys and men. Lancet. 2016;387:1798-9.

90. Giuliano AR, et al. Efficacy of quadrivalent HPV vaccine against HPV Infection and disease in males. $\mathrm{N}$ Engl $\mathrm{J}$ Med. 2011;364(5):401-11.

91. - Joura EA, et al. A 9-valent HPV vaccine against infection and intraepithelial neoplasia in women. $\mathrm{N}$ Engl J Med. 2015;372(8):711-23. Large double-blind, multinational phase $2 b-3$ RCT evaluating $9 v H P V$ vaccine, demonstrating non-inferiority when compared to quadrivalent vaccine $(q H P V)$.

92. Harper DM, et al. Sustained efficacy up to 4.5 years of a bivalent L1 virus-like particle vaccine against human papillomavirus types 16 and 18: follow-up from a randomised control trial. Lancet. 2006;367(9518):1247-55.

93. Joura EA, et al. Efficacy of a quadrivalent prophylactic human papillomavirus (types $6,11,16$, and 18) L1 virus-like-particle vaccine against high-grade vulval and vaginal lesions: a combined analysis of three randomised clinical trials. Lancet. 2007;369(9574):1693-702.

94. Gertig DM, et al. Impact of a population-based HPV vaccination program on cervical abnormalities: a data linkage study. BMC Med. 2013;11:227.

95. Brotherton JM, et al. Early effect of the HPV vaccination programme on cervical abnormalities in Victoria, Australia: an ecological study. Lancet. 2011;377(9783):2085-92.

96. Read TR, et al. The near disappearance of genital warts in young women 4 years after commencing a national human papillomavirus (HPV) vaccination programme. Sex Transm Infect. 2011;87(7):544-7.

97. Ali $\mathrm{H}$, et al. Genital warts in young Australians five years into national human papillomavirus vaccination programme: national surveillance data. BMJ. 2013;346:f2032.

98. de Sanjose S, et al. Human papillomavirus genotype attribution in invasive cervical cancer: a retrospective cross-sectional worldwide study. Lancet Oncology. 2010;11:1048-56.

99. Human papillomavirus vaccines. WHO position paper, October 2014. Wkly Epidemiol Rec. 2014;89(43):465-91.

100. Gotschlich EC, Liu TY, Artenstein MS. Human immunity to the meningococcus. 3. Preparation and immunochemical properties of the group A, group B, and group $\mathrm{C}$ meningococcal polysaccharides. J Exp Med. 1969;129(6):1349-65. 
101. Gotschlich EC, Goldschneider I, Artenstein MS. Human immunity to the meningococcus. IV. Immunogenicity of group $\mathrm{A}$ and group $\mathrm{C}$ meningococcal polysaccharides in human volunteers. J Exp Med. 1969;129(6):1367-84.

102. MacDonald NE, et al. Induction of immunologic memory by conjugated vs plain meningococcal $\mathrm{C}$ polysaccharide vaccine in toddlers: a randomized controlled trial. JAMA. 1998;280(19):1685-9.

103. Pichichero $M$, et al. Comparative trial of the safety and immunogenicity of quadrivalent (A, C, Y, W-135) meningococcal polysaccharide-diphtheria conjugate vaccine versus quadrivalent polysaccharide vaccine in two- to ten-year-old children. Pediatr Infect Dis J. 2005;24(1):57-62.

104. Daugla DM, et al. Effect of a serogroup A meningococcal conjugate vaccine (PsA-TT) on serogroup A meningococcal meningitis and carriage in Chad: a community study [corrected]. Lancet. 2014;383(9911):40-7.

105. Masignani V, et al. Vaccination against Neisseria meningitidis using three variants of the lipoprotein GNA1870. J Exp Med. 2003;197(6):789-99.

106. Public Health England, Meningococcal: the green book in The Green Book. 2015.

107. Santé et Services sociaux Québec. Targeted Meningococcal Serogroup B Vaccination Campaign in the Saguenay-Lac-SaintJean Region. 2016. http://www.msss.gouv.qc.ca/sujets/santepub/ vaccination/index.php?meningococcal-b-vaccination-campaign. Accessed 28 April 2016.

108. Pharmaceutical Benefits Advisory Committee. Public summary document (PSD) July 2015 Pharmaceutical benefits advisory committee meeting. multicomponent meningococcal group $b$ vaccine $(4 \mathrm{cmenb}) ; 0.5 \mathrm{~mL}$ suspension for injection pre-filled syringe; Bexsero ${ }^{\circledR}$ 2015. http://www.pbs.gov.au/info/industry/ listing/elements/pbac-meetings/psd/2015-07/mulit-componentmeningococcal-group-b-vaccine-psd-july-2015. Accessed 28 April 2016.
109. MacNeil JR, et al. Use of Serogroup B Meningococcal Vaccines in Adolescents and Young Adults: recommendations of the Advisory Committee on Immunization Practices. MMWR Morb Mortal Wkly Rep. 2015;64(41):1171-6.

110. Baker CJ, Kasper DL. Correlation of maternal antibody deficiency with susceptibility to neonatal group B streptococcal infection. N Engl J Med. 1976;294(14):753-6.

111. Charles D, Larsen B. Streptococcal puerperal sepsis and obstetric infections: a historical perspective. Rev Infect Dis. 1986;8(3):411-22.

112. Carapetis JR, et al. The global burden of group A streptococcal diseases. Lancet Infect Dis. 2005;5(11):685-94.

113. Steer AC, et al. Status of research and development of vaccines for Streptococcus pyogenes. Vaccine. 2016;34:2953-8.

114. Bialas KM, Swamy GK, Permar SR. Perinatal cytomegalovirus and varicella zoster virus infections: epidemiology, prevention, and treatment. Clin Perinatol. 2015;42(1):61-75.

115. - Henao-Restrepo AM, et al. Efficacy and effectiveness of an rVSV-vectored vaccine expressing Ebola surface glycoprotein: interim results from the Guinea ring vaccination cluster-randomised trial. Lancet. 2015;386(9996):857-66. Interim analysis of ongoing ebola vaccine cluster-randomised trial demonstrating safety and efficacy.

116. Tapia MD, et al. Use of ChAd3-EBO-Z Ebola virus vaccine in Malian and US adults, and boosting of Malian adults with MVA-BN-Filo: a phase 1, single-blind, randomised trial, a phase $1 \mathrm{~b}$, open-label and double-blind, dose-escalation trial, and a nested, randomised, double-blind, placebo-controlled trial. Lancet Infect Dis. 2016;16(1):31-42.

117. World Health Organization. Zika virus and complications. 2016. http://www.who.int/emergencies/zika-virus/en/.

118. National Institute of Allergy and Infectious Diseases. Zika virus vaccine research. 2016. https://www.niaid.nih.gov/topics/Zika/ ResearchApproach/Pages/vaccineResearch.aspx. Accessed 16 April 2016. 